\title{
Brain processes in emotional perception: Motivated attention
}

\author{
Harald T. Schupp, Bruce N. Cuthbert, Margaret M. Bradley, \\ Charles H. Hillman, Alfons O. Hamm, and Peter J. Lang \\ University of Florida, Gainesville, USA
}

\begin{abstract}
Brain potentials and blink reflexes were recorded while participants viewed emotional pictures organised into content categories that varied in motivational significance. Event-related potentials at picture onset showed the largest late positive potentials to erotic scenes and to scenes of threat and mutilation, suggesting heightened attention to contents that are presumed to strongly activate appetitive and defensive motivational systems. Erotic content also showed the greatest sustained attention over the viewing interval as measured by the inhibition of the P3 component of the event-related potential to the late interval startle probe. Among pleasant contents, probe P3 amplitude was inversely related to reported arousal; however, P3 was similarly inhibited across all unpleasant contents. Replicating previous findings, greatest modulation of the startle reflex occurred when participants viewed pictures depicting threat, violent death, and erotica. Overall, the data were seen as consistent with a motivated attention model of emotional perception.
\end{abstract}

Previous studies of brain activity during picture processing have consistently reported large, late positive voltage changes in event-related brain potentials (ERPs) when participants viewed emotional images. This positive waveform, clearly discriminating affective from neutral pictures, begins within a few hundred milliseconds after stimulus onset and may be sustained for several seconds (Cuthbert, Schupp, Bradley, Birbaumer, \& Lang, 2000; Diedrich, Naumann, Maier, \& Becker, 1997; Ilto, Larsen, Smith, \& Cacioppo, 1998;

Correspondence should be addressed to Harald Schupp at the Department of Biological and Clinical Psychology, University of Greifswald, Franz-Mehring-Str. 47, 17487 Greifswald, Germany, or to Margaret Bradley at the Center for the Study of Emotion and Attention, Box 100165, Health Sciences Center, University of Florida, Gainesville, Florida, 32610, USA.

Harald Schupp and Alfons Hamm are at the University of Greifswald. Bruce N. Cuthbert is now at the National Institute of Mental Health. Charles Hillman is now at the University of Illinois. This work was supported in part by National Institute of Mental Health (NIMH) Grants P50 MH52384, MH37757, and MH43975 and by the German Research Foundation (DFG) Grant Schu 1074/7-1.

Konstanzer Online-Publikations-System (KOPS)

URL: http://www.ub.uni-konstanz.de/kops/volltexte/2006/2126/

URN: http://nbn-resolving.de/urn:nbn:de:bsz:352-opus-21261 
Johnston, Miller, \& Burleson, 1986; Keil et al., 2002; Palomba, Angrilli, \& Mini, 1997; Schupp et al., 2000; Schupp, Junghöfer, Weike, \& Hamm, 2003).

Not only are late positive potentials (LPP; Ito et al., 1998) larger when viewing emotional pictures, but their magnitude varies reliably with judged intensity of emotional arousal. Unpleasant pictures rated high in affective arousal are associated with more ERP positivity than unpleasant pictures rated lower in arousal, and this same arousal pattern holds for pleasant pictures (Cuthbert et al., 2000; Schupp et al., 2000). It is suggested that emotionally arousing stimuli activate the brain's motivational circuits, prompting a sustained attentional set that is reflected in the LPP (Bradley, 2000; Lang, Bradley, \& Cuthbert, 1997; Hamm, Schupp, \& Weike, 2003).

The attentional hypothesis has also been explored using a secondary acoustic probe presented during picture viewing. In these experiments, the P3 component of the probe ERP is reliably smaller when viewing pleasant or unpleasant, compared to neutral, pictures (e.g., Schupp, Cuthbert, Bradley, Birbaumer, \& Lang, 1997). This result parallels findings from experiments on instructed attention. When participants are told to attend to one stimulus and ignore others (e.g., Johnson, 1988), a smaller P3 response to a secondary probe is consistently found during the attended stimulus. This result is held to reflect reduced availability of attentional resources for the probe, assuming that the resource pool is limited and there is high allocation to the primary stimulus (Donchin, Kramer, \& Wickens, 1986).

Cuthbert, Schupp, Bradley, McManis, and Lang (1998) found that emotional modulation of the probe P3 wave was similar regardless of whether the secondary probe was an innocuous tone or a sudden, startling stimulus. One advantage of using a startling event as the secondary probe is that it prompts a measurable blink response that is modulated differently from the P3 wave. As numerous studies document, the blink component of the startle reflex is potentiated when viewing unpleasant, and inhibited when viewing pleasant, pictures, and appears to index activation of appetitive or defensive motivational systems (see Bradley, Cuthbert, \& Lang, 1999, for an overview; Lang, 1995). Using a startling stimulus as a secondary probe allows for measurement of both emotional valence with the somatic blink reflex, and attentional resource allocation with the probe ERP.

\section{The research problem}

Previous investigations of picture onset ERPs and probe P3 modulation during picture processing have intermixed pictures from different semantic categories. For instance, arousing pleasant pictures typically include contents, such as erotica, sports, adventure, etc. In a recent study, Bradley, Codispoti, Cuthbert, and Lang (2001a) and Bradley, Codispoti, Sabatinelli, and Lang (2001b) investigated visceral and somatic reactions to specific groups of picture contents 
and found significant pattern differences between these categories. For instance, electrodermal response increase and startle blink inhibition are particularly augmented when participants view pictures of erotica, compared to other arousing pleasant contents, such as sports or adventure. Strong electrodermal reactions and maximum startle potentiation were obtained when viewing pictures of threat or mutilation, compared to other arousing unpleasant contents, such as contamination or accidents. It was concluded that pictures related to primary motivational imperatives - survival of individuals and speciesstrongly activate basic appetitive and defensive systems in the brain, and are thus more likely to drive attention and prompt human emotional experience.

The current study extends this line of investigation to determine how different picture contents modulate voltage potentials from the brain. Whereas previous studies have found differential ERP effects for emotional pictures, we examine here the extent to which these differences are determined by specific picture contents. The pleasant picture categories studied are couples in erotic embrace, attractive opposite sex erotica, romantic couples, and generic and local sports action. Unpleasant contents are scenes involving human threat, animal threat, mutilation, contamination, and loss. Neutral contents are objects and neutral faces. As in Bradley et al. (2001a) each content category includes multiple exemplars. Primary dependent measures in this research are: (1) The ERP to picture onset, particularly the magnitude of the late positive potential (LPP); and (2) the magnitude of the P3 component of the ERP elicited by the secondary startle probe (i.e., the probe P3). To ensure continuity with previous studies, acoustic startle probes are presented during picture viewing and the blink reflex is measured; skin conductance activity is again continuously recorded; ratings of pleasure and arousal are obtained immediately after picture viewing.

\section{Issues and hypotheses}

The onset ERP and probe P3 data were first analysed by averaging across picture content within each valence category (e.g., pleasant, neutral, and unpleasant). Confirmation of the previous general pattern is anticipated: (1) The late positive potential of the onset ERP will be more positive for emotional (pleasant or unpleasant), compared to neutral, pictures; and (2) the probe P3 response will be smaller when viewing emotional (pleasant or unpleasant), compared to neutral, pictures.

The focus of this paper is on whether these measures vary as a function of specific picture content within each valence category. To the extent that a specific picture content is carrying the effect, differences among picture contents within a specific valence category are expected. For example, if the onset ERP is primarily modulated by motivational intensity, it is expected that the late positive potential will be larger when viewing erotica (nude or seminude couples) compared to sports scenes. Pictures of romance-scenes of clothed 
couples kissing and hugging - are explored here for the first time to determine whether motivational effects on the onset ERP (as found for sexually evocative material) might depend on nudity and explicit sexuality. Unpleasant picture contents that imply imminent threat to survival, engage the brain's defence motive system and are expected to markedly modulate onset ERPs. Thus, larger late positive potentials are predicted for pictures depicting threat and mutilated human bodies, when compared with scenes of contamination or loss.

Assuming that the probe P3 reflects the amount of attention allocated to picture processing, its modulation over emotional contents should show a reciprocal pattern to that of the LPP at picture onset - a smaller P3 associated with larger onset positive potentials. It is possible that attention allocation changes over the viewing period, with different contents eliciting a more or less sustained deployment of resources. Early in perceptual processing, resources are required to determine the nature of the content and its motivational meaningfactors that presumably modulate the onset ERP. However, seconds later in the viewing interval, at the time when the probes are delivered, the motivational meaning of the picture stimulus has already been processed and relevant efferent systems activated. Thus, at the point where reflex modulation is most prominent, attentional resources could be differently deployed-reduced, less differentiated, or influenced by other than primary motivational factors. These effects may well vary with semantic contents, and, if so, differences would be reflected in the probe $\mathrm{P} 3$ response.

The overall view taken here is that stimuli with high evolutionary relevance for the survival of individuals and species are most likely to engage the brain's motive systems and to modulate visceral and somatic reflexes. Consistent with previous data, we anticipated that picture contents that are sexually provocative or that imply imminent threat to life will show the most pronounced reactivity. For instance, Bradley et al. (2001) found that pictures of threat elicited the largest blink reflexes and pictures of erotica elicited the smallest, reflexes, whereas both picture contents prompted large skin conductance changes. We hypothesised that the blink response reflects activation of appetitive or defensive motivational systems whereas skin conductance indexes the intensity of motivational activation. We expected to replicate these patterns in the study conducted here.

\section{METHOD}

\section{Participants}

A total of 50 volunteers from the University of Florida Introductory Psychology course participated as part of a class requirement. The data from 10 subjects were omitted from data analysis because of excessive EEG artifacts $(n=5)$, virtually no startle blink response $(n=3)$ or computer failure $(n=2)$ leaving 40 participants (18 female) for the final sample. 


\section{Stimulus materials and design}

Participants viewed 60 pictures from the International Affective Picture System (IAPS; Lang, Bradley, \& Cuthbert, 2001), a collection of standardised photographic materials. ${ }^{1}$ Pictures were chosen to comprise 12 different semantic contents, including 5 pleasant contents (pictures of erotic couples, opposite sex erotica, romantic scenes, generic sport scenes, and locally relevant-i.e., University of Florida-sports scenes $;^{2}$ ) 2 neutral contents (pictures of household objects or neutral human faces); and 5 unpleasant contents (pictures of loss, contamination, mutilations, human threat, animal threat). Each of the 12 picture contents included five different exemplars. Figure 1 illustrates the two dimensional affective space defined by the mean pleasure and arousal ratings for IAPS pictures and specifies the location of the specific picture contents used in this study according to normative ratings of pleasure and arousal.

Pictures were presented on a white matt surface, approximately $2.0 \mathrm{~m}$ from a reclining chair where participants sat. The visual angle of the slides was approximately 24 degrees. Slides were presented for $6 \mathrm{~s}$ using a Kodak Ektagraphic III slide projector and controlled by a Northgate computer. Picture presentation order was balanced across participants. Five different orders of picture presentation were constructed, with each order arranged in blocks of 12, such that there was one exemplar from each of the 12 picture contents in each block of 12. No more than 3 pictures of the same valence was presented consecutively (pleasant, neutral, unpleasant). Across orders, each block was shown once in the first, second, third, fourth, or fifth serial positions. In addition, picture order within blocks was varied such that across participants, each picture was seen equally often in the first or second portion of each block. Each order was presented to approximately one fifth of the participants.

For four of the five exemplars in each picture category, an acoustic startle probe was delivered during picture viewing. The startle probe consisted of a $50 \mathrm{~ms}$ burst of $95 \mathrm{~dB}$ white noise with instantaneous rise time, generated by a Coulbourn S81-02 white noise generator and an S82-24 amplifier. A Quest

\footnotetext{
${ }^{1}$ The International Affective Picture System is available on CD-ROM and as photographic slides. The stimulus sets (CSEA-NIMH, 2001) and technical manual (Lang et al., 2001) can be obtained on request from Margaret Bradley or Peter Lang at the Center for the Study of Emotion and Attention, Box 100165 HSC, University of Florida, Gainesville, FL 32610-0165, USA. IAPS numbers for pictures used in the current study are: Erotic couples, 4651, 4652, 4659, 4664, 4670; Erotic females, 4180, 4250, 4290, 4232, 4310; Erotic males, 4470, 4490, 4520, 4531, 4550; Romance, 4599, 4606, 4609, 4641, 4660; Locally relevant sports, 8111, 8112, 8113, 8114, 8115; Generic sports, 8032, 8034, 8116, 8117, 8220; Neutral objects, 7002, 7009, 7020, 7233, 7235; Neutral faces, 2190, 2200, 2210, 2230, 9070; Loss, 2700, 2800, 2900, 3300, 9421; Contamination, 2730, 7360, 7380, 9300, 9406; Mutilation, 3000, 3053, 3071, 3102, 9405; Animal threat, 1050, 1120, 1201, 1300, 1930; Human threat, $6250,6260,6510,6550,6570$.

${ }^{2}$ Some of the data relating to the local vs. generic sports scenes were previously included in a manuscript that focused solely on sports-related contents (Hillman et al., 2000).
} 


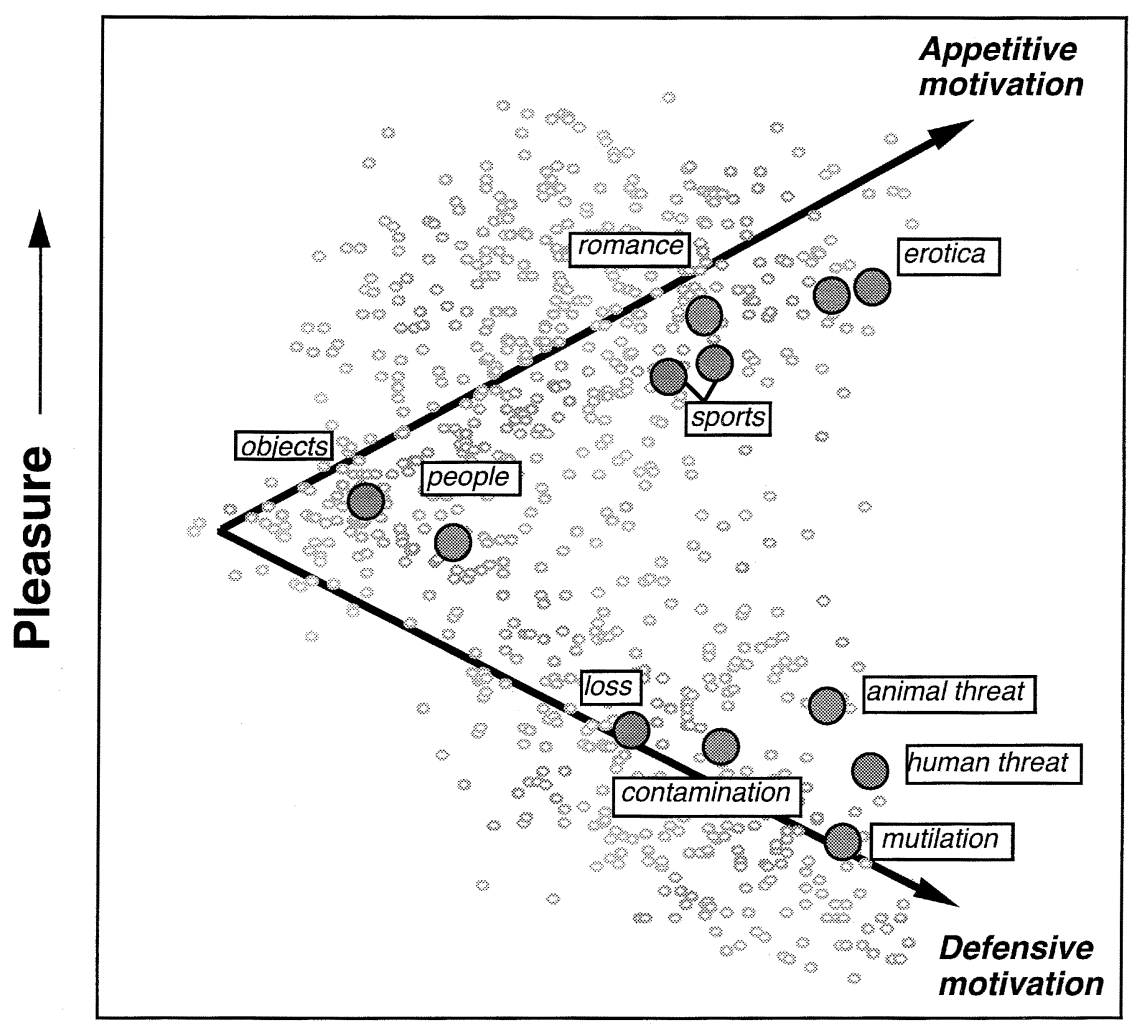

\section{Arousal}

Figure 1. The 2-dimensional affective space defined by the mean pleasure (y-axis) and arousal (x-axis) for IAPS pictures (Lang et al., 2001), and the location of the specific picture contents used in this study, based on the mean pleasure and arousal ratings for the exemplars in each picture content. Vectors indicate the hypothetical appetitive and defensive motivational systems that organise affective evaluations.

model 1700 precision impulse sound level meter was used to calibrate the white noise burst. The probe was delivered at either $2.5 \mathrm{~s}$ or $4.5 \mathrm{~s}$ after picture onset (50/50\% probability). Startle probes were presented binaurally using calibrated Telephonic TDH-49 headphones. Across participants, the noise burst was counterbalanced to ensure that slides were probed during both times equally often. Finally, five startle probes were delivered during the intertrial interval to decrease predictability. 


\section{Apparatus and response measures}

The electroencephalogram (EEG) was recorded using a Nihon-Kohden amplifier and LabView software on a Macintosh computer. Data were continuously recorded during a $10 \mathrm{~s}$ epoch that included $2 \mathrm{~s}$ prior to picture onset, $6 \mathrm{~s}$ of picture presentation, and $2 \mathrm{~s}$ following picture offset. The International 10-20 system was followed for seven electrode sites: F3, Fz, F4, Cz, P3, Pz, and P4; in addition, Sensormedics silver/silver chloride miniature electrodes were placed on the mastoids (A1, A2). All channels were referenced to $\mathrm{Cz}$ and digitally rereferenced offline to linked mastoids. Vertical and horizontal eye movements were recorded using Sensormedics silver/silver chloride miniature electrodes to account for ocular artifacts. A $35 \mathrm{~Hz}$ high frequency cut-off and a $10 \mathrm{~s}$ time constant $(0.016 \mathrm{~Hz}$ low frequency-cut off) were used to record all cortical and ocular channels. To shorten the length of time between EEG channels, the data sampling rate was $1250 \mathrm{~Hz} /$ channel, and converted offline to $125 \mathrm{~Hz} / \mathrm{channel}$, by discarding 9 out of every 10 samples. To correct for vertical and horizontal ocular artifacts, an eye movement artifact correction procedure (Gratton, Coles, \& Donchin, 1983; Miller, Gratton, \& Yee, 1988) was applied to the EEG recording separately for the picture onset EEG and the startle probe EEG.

Stimulus presentation, stimulus timing, and collection of other physiological data (e.g., skin conductance, blink responses, corrugator EMG activity, and evaluative judgements) were controlled by a Northgate 486 microcomputer running VPM software (Cook, 1994).

Skin conductance was collected from adjacent sites with standard $(8.8 \mathrm{~mm})$ Sensormedics silver/silver chloride electrodes placed on the hypothenar eminence of the left palm. The electrodes were filled with 0.05 Molar Unibase cream electrolyte. A Coulbourn S71-22 skin conductance coupler administered a constant 0.5 volts across sensors. The skin conductance coupler was calibrated to record a range of $0-40 \mu$ siemens before each recording session.

The blink response was measured by recording EMG activity over the orbicularis oculi muscle beneath the left eye, using two adjacent Sensormedics silver/silver chloride miniature electrodes filled with electrolyte paste. The raw EMG signal was amplified and routed through a Coulbourn S75-01 bioamplifier. The signal was bandpass filtered from $90 \mathrm{~Hz}$ to $250 \mathrm{~Hz}$. It was then rectified and integrated using a Coulbourn S76-01 contour follower with a time constant of $125 \mathrm{~ms}$. The blink response was sampled at $1000 \mathrm{~Hz}$ from $50 \mathrm{~ms}$ before until $250 \mathrm{~ms}$ after the acoustic startle probe onset.

Evaluative judgements of pleasure and arousal were measured using the SelfAssessment Manikin (SAM: Lang, 1980). SAM is an animated, interactive computer display that acquires ratings of pleasure and arousal on 0 to 20 point scale and is controlled by an IBM-XT computer running VPM data acquisition software (Cook, 1994). SAM was displayed on a 12 inch computer monitor 
approximately $2.0 \mathrm{~m}$ from the participant directly below the slide projection screen.

\section{Procedure}

On completion of informed consent, physiological sensors were attached. Participants were instructed that a slide would appear on the screen in front of them and that each picture should be viewed the entire time $(6 \mathrm{~s})$ it was on the screen. At picture offset, the SAM figure appeared on the ratings monitor and evaluative judgements were measured. An intertrial interval varying from $6 \mathrm{~s}$ to $18 \mathrm{~s}$ occurred after the ratings. Participants were instructed that occasional noises heard over headphones could be ignored. Following all picture trials, participants filled out a number of post-experimental questionnaires and were given credit, debriefed, and thanked.

\section{Data reduction}

Picture viewing. All individual EEG trials that were off-scale at any time during the $10 \mathrm{~s}$ recording epoch $(2 \mathrm{~s}$ pre-picture onset, $6 \mathrm{~s}$ picture duration, $2 \mathrm{~s}$ post-picture offset) were excluded from further analysis. Following eye movement correction, grand average waveforms were calculated separately for each picture category and electrode site, and referenced to a $120 \mathrm{~ms}$ baseline preceding picture onset. The general topography of the ERP waveform included evoked potential components followed by a sustained positive slow wave. Following previous studies (cf. Cuthbert et al., 2000), the late positive potential was assessed using an area measure lasting from $400 \mathrm{~ms}$ to $700 \mathrm{~ms}$. Further development of the positive wave was assessed using a second area measure in a time window lasting from $700 \mathrm{~ms}$ to $1000 \mathrm{~ms}$.

Skin conductance responses were expressed as the maximum change occurring between $1 \mathrm{~s}$ and $4 \mathrm{~s}$ after picture onset, relative to a 1 second prepicture baseline period. As is typical for skin conductance data, the distribution of change scores was skewed (skewness $=4.45$ ) and a Kolmogorov-Smirnov test indicated a significant deviation from normality $(d=.33, p<.001)$; accordingly a $\log$ transformation $[\log (\mathrm{SCR}+1)]$ was applied to normalise the data (Venables \& Christie, 1980).

Probe processing. Separate epochs of stimulus-synchronised EEG traces were extracted from the whole $10 \mathrm{~s}$ EEG recording, extending from $120 \mathrm{~ms}$ before until $1 \mathrm{~s}$ after probe onset. The P3 wave of the ERP was scored by determining the base-to-peak amplitudes on averaged waveforms for each subject, picture category, and electrode site. ${ }^{3}$ The P3 component was scored

\footnotetext{
${ }^{3}$ Previous studies investigating the ERP to a startle probe (e.g., Cuthbert et al., 1998; Schupp et al., 1997) found no differences as a function of picture valence prior to $300 \mathrm{~ms}$. Thus, in the current study, we focused on a time window that encompassed the P3 response.
} 
within a window beginning at N2 latency and continuing until $504 \mathrm{~ms}$. When an unequivocal positive peak could not be detected, an inflection point was calculated such that the smallest vertical distance between adjacent points within the time window defined the location for the latency of the P3, with the corresponding amplitude as the peak score.

The blink data were analysed offline using a peak scoring algorithm developed by Globisch, Hamm, Schneider, and Vaitl (1993), which scores each trial for amplitude in arbitrary A/D units and onset latency in ms. Peak magnitude was detected in a $20-150 \mathrm{~ms}$ time window after startle probe onset. The raw blinks were standardised within subjects using a $z$-transformation (i.e., raw scores for each subject were deviated from the individual's mean score and divided by his/ her standard deviation) to decrease variability due to differences in the absolute size of the startle blink across subjects, and expressed as $T$-scores [i.e. $(z \times 10)+$ 50], which produces a mean of 50 and a standard deviation of 10 .

\section{ERP data analyses}

Initial analyses of the ERP data included the factor Affect (pleasant, neutral, and unpleasant) and Site $(\mathrm{Fz}, \mathrm{Cz}, \mathrm{Pz})$. Replicating previous effects, affective modulation was greatest at the parietal sensor site for both of the onset ERP measures, Affect $\times$ Site $F \mathrm{~s}(4,36)>5.3, p<.01$, as well as for the probe P3, Affect $\times$ Site $F(4,36)=4.9, p<.01$. Accordingly, for brevity, ERP analyses for specific picture contents included parietal leads only.

To assess differences among specific contents within each valence category, the analytic plan involved separate analyses for the 5 pleasant, 5 unpleasant, and 2 neutral picture contents. Two analyses were conducted for both sets of ERP data, including: (1) analysis of Pz; and (2) an analysis of left and right parietal leads, which used an additional Laterality factor. For factors with more than two repeated measurement levels, a multivariate test statistic (Wilks' lambda) was used as error inflation control. For ANOVAs that produced a significant main effect of picture content, pairwise comparisons were subsequently conducted using a Bonferroni adjustment.

Previous research (Bradley et al., 2001b) suggests that a subdivided sample would not have sufficient power for reliably assessing sex differences. Consistent with this assumption, the primary analyses including sex as a factor did not yield interactions or main effects at an acceptable $p$-value. In all cases, the general direction of effects was similar for males and females. The data reported are for the participant sample as a whole.

\section{RESULTS}

Picture onset ERPs. Table 1 lists the mean responses for pleasant, neutral, and unpleasant pictures. When averaged across specific picture content, onset ERPs differed as a function of emotional valence in both the $400-700 \mathrm{~ms}$ region and in the $700-1000 \mathrm{~ms}$ region, $F_{\mathrm{s}}(2,38)>14.0, p<.001$, replicating previous 
TABLE 1

Mean ERP onset area measures (400-700 ms and 700-1000 ms), probe P3 responses, and other dependent measures when viewing pleasant, neutral, and unpleasant pictures, averaged across specific content (SDs in parentheses)

\begin{tabular}{lccccc}
\hline Dependent measure & \multicolumn{2}{c}{ Pleasant } & \multicolumn{2}{c}{ Neutral } & \multicolumn{2}{c}{ Unpleasant } \\
\hline Onset ERP, Pz, 400-700 ms $(\mu \mathrm{V})$ & 12.4 & $(5.6)$ & 7.7 & $(6.8)$ & $9.0(5.9)$ \\
Onset ERP, Pz, 700-1000 ms $(\mu \mathrm{V})$ & 14.0 & $(5.6)$ & 9.0 & $(7.1)$ & $12.8(6.9)$ \\
Probe P3, Pz $(\mu \mathrm{V})$ & 13.9 & $(7.7)$ & 20.2 & $(10.1)$ & $15.7(8.3)$ \\
Blink magnitude $(T$-score) & $47.8(1.5)$ & 48.7 & $(2.6)$ & $50.8(2.1)$ \\
Skin conductance peaks (log $\mu$ siemens) & $0.06(0.07)$ & $0.04(0.07)$ & $0.06(0.09)$ \\
Pleasure ratings $(0-20$ scale) & $13.5(3.2)$ & 9.7 & $(2.2)$ & $5.2(2.9)$ \\
Arousal ratings $(0-20$ scale) & $12.4(3.1)$ & 5.8 & $(3.1)$ & 12.9 & $(3.3)$ \\
\hline
\end{tabular}

findings (e.g., Cuthbert et al., 2000). Pleasant pictures prompted larger late positive potentials than did viewing neutral materials in both windows, $F_{\mathrm{s}}(1,39)$ $>21, p<.001$, and unpleasant pictures prompted larger positive potentials in the $700-1000 \mathrm{~ms}$ window compared to neutral pictures, $F(1,39)=17.5, p<.001$.

Figure 2 illustrates the onset ERP waveforms for each of the specific picture contents, and Table 2 lists the area measures for each picture content. For pleasant contents, erotic pictures involving couples or opposite sex erotica,

\section{Picture Onset ERPs (Pz)}
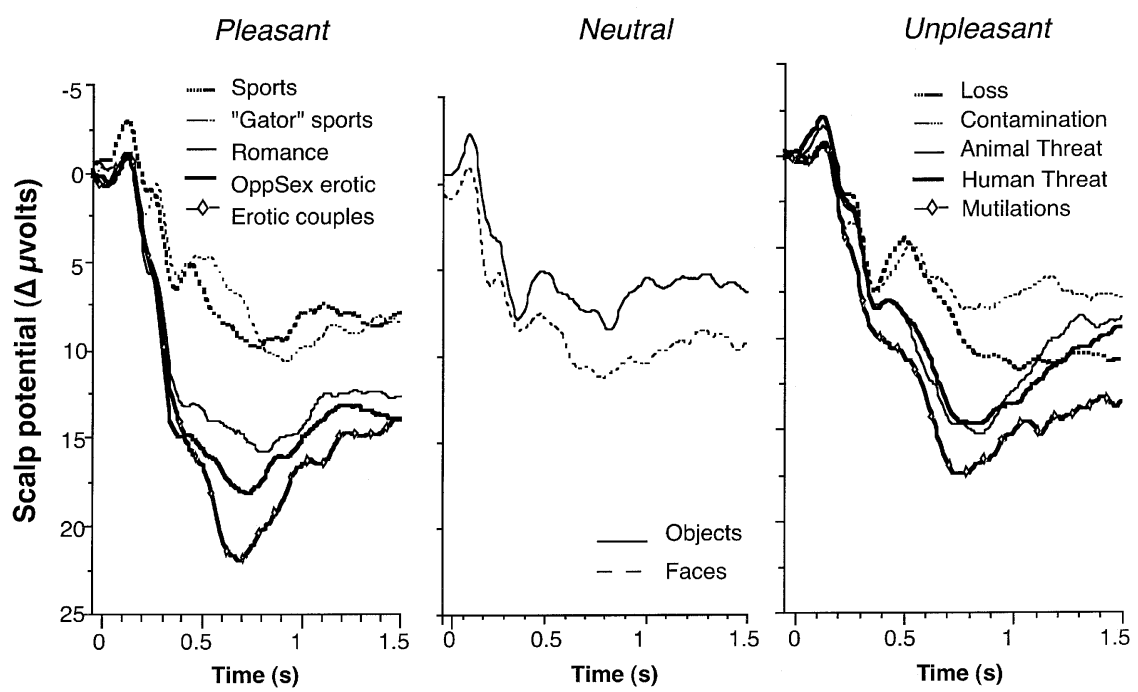

Figure 2. Stimulus-synchronised grand average ERP waveforms for the Pz sensor during viewing of specific pleasant, neutral, and unpleasant pictures. 
TABLE 2

Mean ERP onset area measures (400-700 and 700-1000 ms) when viewing specific pleasant (top), neutral (middle), and unpleasant (bottom) picture contents ( $S D$ s in parentheses)

\begin{tabular}{lcc}
\hline Picture content & $\begin{array}{c}\text { Pz-onset ERP } \\
(400-700 \mathrm{~ms})^{\mathrm{a}}\end{array}$ & $\begin{array}{c}\text { Pz-onset ERP } \\
(700-1000 \mathrm{~ms})^{\mathrm{a}}\end{array}$ \\
\hline Erotic couples & $18.6(8.1) \mathrm{a}$ & $19.4(9.6) \mathrm{a}$ \\
Opposite sex erotica & $16.3(8.4) \mathrm{a}, \mathrm{b}$ & $16.8(7.6) \mathrm{a}, \mathrm{b}$ \\
Romance & $13.8(7.2) \mathrm{b}$ & $15.2(7.8) \mathrm{b}$ \\
Local sports & $7.3(7.4) \mathrm{c}$ & $9.4(7.1) \mathrm{c}$ \\
Generic sports & $5.5(6.9) \mathrm{c}$ & $9.7(6.3) \mathrm{c}$ \\
\hline Neutral objects & $6.0(7.7) \mathrm{a}$ & $7.0(8.5) \mathrm{a}$ \\
Neutral faces & $9.3(8.5) \mathrm{b}$ & $10.8(8.6) \mathrm{b}$ \\
\hline Human threat & $9.9(6.8) \mathrm{A}, \mathrm{B}$ & $14.3(7.7) \mathrm{A}, \mathrm{C}$ \\
Animal threat & $10.5(7.9) \mathrm{A}$ & $14.5(9.8) \mathrm{A}$ \\
Mutilation & $12.5(7.7) \mathrm{A}$ & $16.7(9.7) \mathrm{A}$ \\
Contamination & $6.0(8.2) \mathrm{B}, \mathrm{C}$ & $8.1(8.7) \mathrm{B}$ \\
Loss & $6.1(7.9) \mathrm{C}$ & $10.6(8.3) \mathrm{B}, \mathrm{C}$ \\
\hline
\end{tabular}

\footnotetext{
Note: within each valence category (e.g., pleasant, neutral, unpleasant) and each measure, contents that share at least one letter are not significantly different in pairwise comparisons $(p<.005)$. ${ }^{a}$ Area measure in the specified window $(\mu \mathrm{V})$.
}

elicited significantly more positivity for both area measures relative to sports displays, content $F \mathrm{~s}(4,36)>20, p<.0001$. Pictures of romantic couples also evoked sizeable positive slow waves that were similar in magnitude to those elicited when viewing pictures of opposite sex erotica. Effects at lateral leads were identical to those obtained at Pz.

For the unpleasant picture contents, a main effect of picture content was found for both area measures, content $F \mathrm{~s}(4,36)>8.4, p<.001$. Pictures depicting mutilation and animal threat elicited significantly more positivity than did pictures of loss or contamination for both area measures (see Table 2). Pictures of human threat also elicited pronounced positive slow waves that were similar in magnitude to those elicited when viewing pictures of mutilations and animal threat. Analyses of the lateral leads indicated that pictures of mutilations prompted significantly greater positivity than all other picture contents.

Startle probe P3 amplitude. Replicating previous findings (e.g., Schupp, Cuthbert, Bradley, Birbaumer, \& Lang, 1997), the P3 response to the startle probe was significantly modulated by affective valence, $F(2,38)=12.2, p<.01$. Pleasant and unpleasant pictures elicited smaller probe P3s compared to neutral images, $F_{\mathrm{S}}(1,39)>15, p<.01$. In addition, pleasant pictures elicited slightly 
smaller probe $\mathrm{P} 3 \mathrm{~s}$ compared to unpleasant contents, $F(1,39)=4.6, p<.05$ (see Table 1).

Figure 3 (top) illustrates probe P3 amplitude as a function of specific picture content. For pleasant picture contents, modulation of the P3 component of the probe ERP showed a slightly different pattern of modulation than did the onset ERPs, content $F(4,36)=11.1, p<.001$. Whereas scenes of erotic couples and opposite sex erotica prompted the smallest probe P3 amplitudes, the probe P3 when viewing these explicitly erotic materials differed from less sexually explicit pictures of romance, $F \mathrm{~s}(1,39)>10, p<.01$. Moreover, whereas pictures of generic and local sports scenes prompted equivalent onset ERPs, pictures of the more personally relevant local sports led to a significantly smaller probe P3 response, compared to when viewing generic sports teams, $F(1,39)=9.5, p<$ .01 . Analyses of P3 and P4 leads confirmed the analysis of the midline leads, and resulted in no significant interactions involving laterality.

For unpleasant pictures, modulation of the P3 component of the probe ERP also showed a different pattern than did the onset ERPs. Whereas onset ERPs were most pronounced for pictures of threat and mutilation, differential P3 modulation as a function of specific content was not strong, and did not reach significance, $F(4,36)=2.4, p=.07$. Thus, viewing unpleasant pictures was associated with a significantly smaller P3 response, compared to neutral pictures, and this effect was fairly similar across different unpleasant contents. The analyses of P3 and P4 leads confirmed the analysis of the midline leads, and indicated no significant interaction involving laterality.

Startle blink magnitude. As expected, when averaged over specific picture contents, the magnitude of the startle blink response was significantly modulated by affective valence, $F(2,38)=21.6, p<.001$, with larger blinks elicited when viewing unpleasant (see Table 1) compared to pleasant, $F(1,39)=42.0, p<$ .001 , or neutral pictures, $F(1,39)=11.7, p<.001$. Pleasant pictures prompted smaller blinks than neutral pictures, $F(1,39)=4.7, p<.05$.

Figure 3 (bottom) illustrates blink magnitude for pictures as a function of specific content. For pleasant pictures, a main effect of stimulus content, $F(4,36)=5.2, p<.05$, and pairwise comparisons replicated the finding that erotic contents (erotic couples or opposite sex erotica) prompted the most blink inhibition and did not significantly differ from one another (Bradley et al., 2001a). Importantly, pictures involving less sexually explicit pictures (of romance) also prompted small reflexes that did not differ from those elicited when viewing the more explicitly erotic materials. Pictures of generic sports and local sports prompted significantly larger blink reflexes than did pictures of erotica and did not significantly differ from each other.

For unpleasant pictures, a main effect of picture content $F(4,36)=8.1, p<$ .01 , and pairwise comparisons again replicated the pattern reported previously (Bradley et al., 2001a). The largest blink reflexes were elicited when viewing 


\section{Startle probe P3}
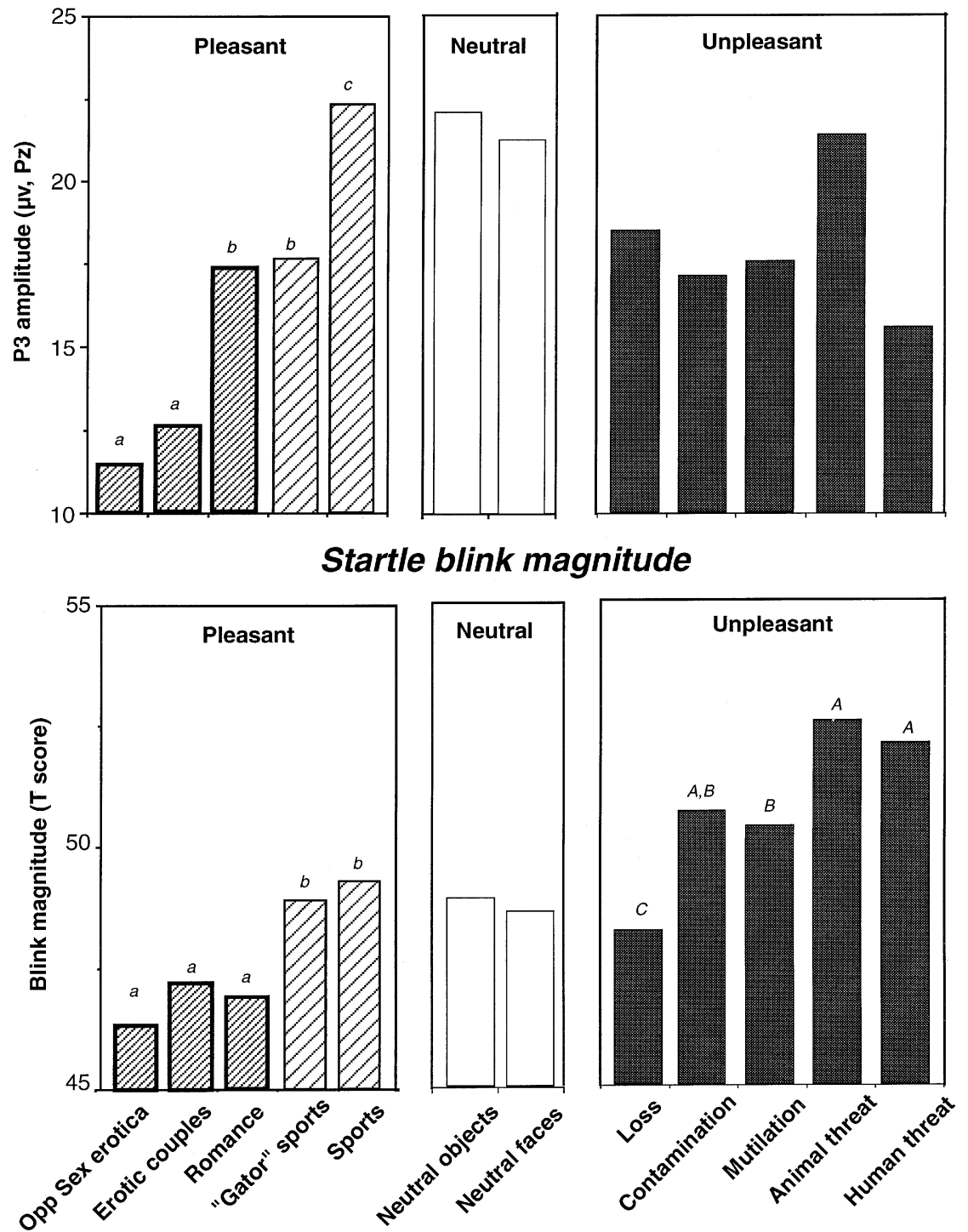

Figure 3. Top panel: Magnitude of the $\mathrm{P} 3$ response to a startle probe for specific pleasant, neutral, and unpleasant contents. Within a valence category, specific picture contents that share any letters are not significantly different. Bottom panel: The magnitude of the reflexive blink response to a startle probe for specific pleasant, neutral, and unpleasant contents. 
pictures displaying human or animal threat. These were slightly, but significantly larger than those elicited when viewing pictures of mutilations. Pictures of contamination prompted relatively potentiated blink responses, and all of these contents prompted larger blinks than pictures of loss.

Picture processing: Skin conductance and evaluative judgements. Table 3 lists the mean pleasure and arousal ratings, and peak skin conductance changes as a function of specific picture content, as well as the outcome of pairwise comparisons for each measure.

In general, all of the pleasant contents were rated similarly high in pleasantness, with only generic sports pictures rated slightly lower in pleasure than each of the other contents, content $F(4,36)=4.2, p<.01$. Erotic pictureseither couples or opposite sex erotica-were rated as more arousing than pictures of romance or either type of sports scenes, content $F(4,36)=13.4, p<$ .001 . Skin conductance changes paralleled arousal ratings, content $F(4,36)=$ $7.4, p<.01$, with both types of erotic stimuli prompting larger changes than pictures of romance or sports, which did not differ.

For unpleasant contents, slight differences in affective valence ratings indicated that pictures of mutilations were rated as most unpleasant and pictures of

TABLE 3

Mean pleasure and arousal ratings, and skin conductance changes when viewing specific pleasant (top), neutral (middle), and unpleasant (bottom) picture contents ( $S D$ s in parentheses)

\begin{tabular}{lccc}
\hline Picture content & Pleasure ratings $^{\mathrm{a}}$ & Arousal ratings $^{\mathrm{a}}$ & $S^{\mathrm{b}} \mathrm{CR}^{\mathrm{b}}$ \\
\hline Erotic couples & $14.2(3.9) \mathrm{a}$ & $14.4(4.2) \mathrm{a}$ & $0.10(0.11) \mathrm{a}$ \\
Opposite sex & $14.1(4.0) \mathrm{a}$ & $13.8(4.5) \mathrm{a}$ & $0.08(0.09) \mathrm{b}$ \\
Erotica & $13.3(3.6) \mathrm{a}$ & $11.4(3.5) \mathrm{b}$ & $0.03(0.06) \mathrm{c}$ \\
Romance & $13.2(3.5) \mathrm{a}$ & $11.5(3.5) \mathrm{b}$ & $0.04(0.07) \mathrm{c}$ \\
Local sports & $12.5(3.1) \mathrm{b}$ & $10.9(2.9) \mathrm{b}$ & $0.03(0.07) \mathrm{c}$ \\
Generic sports & $10.1(2.7) \mathrm{a}$ & $4.9(3.7) \mathrm{a}$ & $0.03(0.07)$ \\
\hline Neutral objects & $9.3(1.9) \mathrm{b}$ & $6.6(3.6) \mathrm{b}$ & $0.04(0.09)$ \\
Neutral faces & $4.9(3.7) \mathrm{B}$ & $14.7(4.2) \mathrm{A}$ & $0.08(0.10) \mathrm{B}$ \\
\hline Human threat & $6.0(3.3) \mathrm{C}$ & $13.8(3.6) \mathrm{B}$ & $0.06(0.10) \mathrm{B}$ \\
Animal threat & $3.8(3.5) \mathrm{A}$ & $13.9(4.2) \mathrm{B}$ & $0.11(0.15) \mathrm{A}$ \\
Mutilation & $5.3(3.2) \mathrm{B}, \mathrm{C}$ & $11.8(3.6) \mathrm{C}$ & $0.03(0.08) \mathrm{C}$ \\
Contamination & $5.6(2.5) \mathrm{B}, \mathrm{C}$ & $10.1(3.7) \mathrm{D}$ & $0.04(0.06) \mathrm{C}$ \\
Loss &
\end{tabular}

Note: Within each valence category (e.g., pleasant, neutral, unpleasant) and each measure, contents that share at least one letter are not significantly different in pairwise comparisons. ${ }^{\mathrm{a}} 0-20$ rating scale. ${ }^{\mathrm{b}}$ Peak skin conductance change ( $\mu$ siemens; log transform). 
animal threat rated as least unpleasant, content $F(4,36)=8.3, p<.001$. Threat and mutilation pictures were rated as more arousing than pictures of contamination or loss, and pictures of loss were rated as least arousing content, $F(4,36)=14.0, p<.001$. Paralleling the arousal ratings, skin conductance responses, content $F(4,36)=4.4, p<.01$, were larger when viewing threat (either animal or human) and mutilations compared to pictures of contamination or loss.

Neutral pictures. Neutral faces were rated as slightly less pleasant, $F(1,39)$ $=6.1, p<.05$, and more arousing than household objects, $F(1,39)=9.6, p<.02$ (see Table 1, middle panel) but elicited equivalent skin conductance changes and were not different in terms of probe P3 amplitude or blink magnitude (see Figure $3), F \mathrm{~s}(1,39), p<1.2$, n.s. Neutral faces elicited slightly, but significantly, more positivity at picture onset for both area measures, consistent with a significant difference between these contents in ratings of arousal, $F(1,39)=5.0$ and $6.1, p$ $<.05$ (see Table 1).

\section{DISCUSSION}

Replicating previous studies, differences in the amplitude of the late positive potential of the ERP elicited at picture onset as well as the magnitude of the P3 response to a secondary probe varied as a function of the emotional content of pictures. Larger LPPs were obtained at picture onset for emotional (pleasant or unpleasant) compared to neutral pictures, and smaller probe P3 waves were found when viewing emotional compared to neutral pictures. The reciprocal relationship between these two measures prompted the hypothesis that both index attentional engagement. That is, if more attentional resources are allocated to motivationally relevant cues, one expects less to be available for processing the secondary startle probe. Finding a larger late positive potential at picture onset and a smaller probe $\mathrm{P} 3$ for emotional, compared to neutral, pictures is consistent with this hypothesis. The current study provides a window onto the brain processes occurring when humans perceive specific pleasant and unpleasant picture contents, and sheds additional light on the reciprocal attention hypothesis.

Pleasant pictures. Among pleasant contents, erotic pictures prompted the largest late positive potentials as well as the smallest probe P3 responses. Moreover, these materials also occasioned the largest skin conductance changes, and the most inhibited blink reflexes, replicating effects reported previously (Bradley et al., 2001a). We interpret these data as indicating that sexually explicit stimuli naturally activate the appetitive motivational system, capturing and sustaining attentional processing.

Like erotic materials, pictures of romance also prompted large late positive potentials at picture onset and inhibited blink reflexes, implying a parallel 
motivationally determined selective attention. On the other hand, the probe P3 response was significantly less inhibited when viewing romantic compared to erotic pictures, implying an attentional difference later in the viewing interval. These data suggest that while the less sexually explicit, romantic content captured attention and activated efferent systems (e.g., the startle blink), perceptual processing of this content was less sustained.

Probe P3 also indexed late interval processing differences in the sport contents. Whereas general and local sports pictures prompted similar onset ERPs and equivalent blink reflexes, the probe P3 was significantly more inhibited during local sports scenes than for generic sports content. That is, pictures expected to be of interest to local fans prompted more sustained attention. Nevertheless, sports scenes, overall, did not strongly engage basic motive systems in the brain. Compared to the sexually related contents, sports content showed modest onset ERPs and little blink inhibition.

Unpleasant pictures. Pictures depicting threat and mutilation prompted the largest late positive potentials in the onset ERP. These materials also occasioned the largest skin conductance changes and the greatest blink potentiation of all unpleasant contents. From an evolutionary perspective, pictures depicting violence and death are expected to rapidly and strongly engage the brain's defence motive system, capturing attention and mediating defensive reflexes. Less imminent, more remote or more defensible threats (pictures of contamination or loss) show less pronounced motivational engagement and efferent activation.

Interestingly, the probe P3 response was generally inhibited for all unpleasant pictures, compared to neutral stimuli, and this inhibition did not vary with content. This result suggests that aversive cues in general have a significant, sustained call on attentional resources. Bradley et al. (2001a) came to a similar conclusion, based on the finding of a consistent, prolonged cardiac deceleration during the viewing of all the unpleasant picture contents tested in their experiment. The meaning of aversion's broad attentional call is not clear. However, animal biologists (e.g., Blanchard \& Blanchard, 1989; Timberlake, 1993) have frequently noted that immobility and information gathering are initial responses to threat at a distance. Conceivably, this state is maintained until the provocation is removed, or its approach requires overt action. Lang et al. (1997) have described the sequencing of the psychophysiological responses that characteristically occur in response to threat (i.e., "the defense cascade"), and noted that this pattern is replicated when humans view unpleasant stimuli. Initial responses to low arousal stimuli (threat at a distance) are related to attention-cardiac deceleration and inhibition of both the startle probe reflex and the probe P3. Startle potentiation occurs in the context of larger onset ERPs to stimuli of high motivational relevance. These later cues (direct threat to the viewer) imply greater imminence of harm- 
stimulus events closer to the point where information gathering must give way to defensive action.

Conclusions. The results encourage several conclusions about emotional picture processing. The EEG response is clearly most pronounced to contents of sex and violence that engage motivational circuits in the brain presumably related to the survival of individuals and species. It is provocative that the BOLD response when viewing pictures shows the same pattern-more extensive and stronger activation for pictures of sex and violence (Bradley et al., 2003). For these arousing picture stimuli, the primary response is attention-a close scrutiny that may continue long into the period of picture exposure. This mobilisation of attention is apparently the same as that occasioned by instruction (e.g., look at stimulus A and ignore stimulus B), or that is prompted spontaneously by experience or interest (e.g., the sports fan's reaction to his team). The data also suggests that the brain may be more broadly attentive to a broad range of aversive, compared to pleasant stimuli, in the sense that resources are allocated even to cues that are low in arousal or threat imminence. Like the motorist who slows to closely watch the scene of an accident, attention is drawn to and sustained by all signs of threat or harm. In a larger sense, these findings make it clear that understanding ERP activity during emotional perception will depend on knowing the relationship of these potentials to the complex array of behavioural, evaluative, and peripheral physiological responses deployed by the brains appetitive and defensive motive systems.

Manuscript received 6 December 2001 Revised manuscript received 3 February 2003

\section{REFERENCES}

Blanchard, R. J., \& Blanchard, D. C. (1989). Attack and defense in rodents as ethoexperimental models for the study of emotion. Progress in Neuro-Psychopharmacological \& Biological Psychiatry, 13, 3-14.

Bradley, M. M. (2000). Emotion and motivation. In J. T. Cacioppo, L. G. Tassinary, \& G. Berntson (Eds.), Handbook of psychophysiology (pp. 602-642). New York: Cambridge University Press.

Bradley, M. M., Codispoti, M., Cuthbert, B. N., \& Lang, P. J. (2001a). Emotion and motivation: I. Defensive and appetitive reactions in picture processing. Emotion, 1, 276-298.

Bradley, M. M., Codispoti, M., Sabatinelli, D., \& Lang, P. J. (2001b). Emotion and motivation: II. Sex differences in picture processing. Emotion, 1, 300-319.

Bradley, M. M., Cuthbert, B. N., \& Lang, P. J. (1999). Affect and the startle reflex. In M. E. Dawson, A. Schell, \& A. Boehmelt (Eds.), Startle modification: Implications for neuroscience, cognitive science and clinical science (pp. 157-183). Stanford, CA: Cambridge University Press.

Bradley, M. M., Sabatinelli, D., Lang, P. J., Fitzsimmons, J. R., King, W. M., \& Desai, P. (2003). Activation of the visual cortex in motivational attention. Behavioral Neuroscience, 117, 369-380.

Center for the Study of Emotion and Attention (CSEA-NIMH) (1999). The international affective picture system: Digitized photographs. Gainesville, FL: The Center for Research in Psychophysiology, University of Florida. 
Cook, E. W. I. (1994). VPM reference manual. Department of Psychology, University of Alabama, Birmingham, AL.

Cuthbert, B. N., Schupp, H. T., Bradley, M. M., Birbaumer, N., \& Lang, P. J. (2000). Brain potentials in affective picture processing: Covariation with autonomic arousal and affective report. Biological psychology, 52, 95-111.

Cuthbert, B. N., Schupp, H. T., Bradley, M. M., McManis, M. H., \& Lang, P. J. (1998). Probing affective pictures: Attended startle and tone probes. Psychophysiology, 35, 344-347.

Diedrich, O., Naumann, E., Maier, S., \& Becker, G. (1997). A frontal positive slow wave in the ERP associated with emotional slides. Journal of Psychophysiology, 11, 71-84.

Donchin, E., Kramer, A. F., \& Wickens, C. (1986). Applications of brain event-related potentials to problems in engineering psychology. In M. G. H. Coles, E. Donchin, \& S. W. Porges (Eds.), Psychophysiology (pp. 702-718). New York: Guilford Press.

Globisch, J., Hamm, A., Schneider, R., \& Vaitl, D. (1993). A computer program for scoring reflex eyeblink and electrodermal responses written in Pascal. Psychophysiology, 39, S30.

Gratton, G., Coles, M. G., \& Donchin, E. (1983). A new method for off-line removal of ocular artifact. Electroencephalography and Clinical Neurophysiology, 55, 468-484.

Hamm, A. O., Schupp, H. T., \& Weike, A. I. (2003). Motivational organization of emotions: Autonomic changes, cortical responses, and reflex modulation. In R. J. Davidson, K. Scherer, \& H. H. Goldsmith (Eds.), Handbook of affective sciences (pp. 187-211). Oxford, UK: Oxford University Press.

Hillman, C. H., Cuthbert, B. N., Cauraugh, J., Schupp, H. T., Bradley, M. M., \& Lang, P. J. (2000). Psychophysiological responses of sport fans. Motivation and Emotion, 24, 13-28.

Ito, T. A., Larsen, J. T., Smith, N. K., \& Cacioppo, J. T. (1998). Negative information weighs more heavily on the brain: The negativity bias in evaluative categorizations. Journal of Personality and Social Psychology, 75, 887-900.

Johnson, R. J. (1988). The amplitude of the P300 component of the event-related potential: Review and synthesis: In P. K. Ackles, J. R. Jennings, \& M. G. H. Coles (Eds.), Advances in psychophysiology (Vol.3, pp. 69-137). Greenwich, CT: JAI Press.

Johnston, V. S., Miller, D. R., \& Burleson, M. H. (1986). Multiple P3s to emotional stimuli and their theoretical significance. Psychophysiology, 23, 684-693.

Keil, A., Bradley, M. M., Hauk, O., Rockstroh, B., Elbert, T. R., \& Lang, P. J. (2002). Large-scale neural correlates of affective picture viewing. Psychophysiology, 39, 641-649.

Lang, P. J. (1980). Behavioral treatment and the bio-behavioral assessment: Computer applications. In J. B. Sidowski, J. H. Johnson, \& T. A. Williams (Eds.), Technology in mental health care delivery systems (pp. 119-137). Norwood, NJ: Ablex.

Lang, P. J. (1995). The emotion probe: Studies of motivation and attention. American Psychologist, 50, 371-385.

Lang, P. J., Bradley, M. M., \& Cuthbert, B. N. (1997). Motivated attention: Affect, activation, and action. In P. J. Lang, R. F. Simons, \& M. Balaban (Eds.), Attention and orienting (pp. 97-135). Mahwah, NJ: Erlbaum.

Lang, P. J., Bradley, M. M., \& Cuthbert, B. N. (2001). International affective picture system (IAPS): Instruction manual and affective ratings. Technical Report A-5, The Center for Research in Psychophysiology, University of Florida.

Miller, G. A., Gratton, G., \& Yee, C. M. (1988). Generalized implementation of an eye movement correction procedure. Psychophysiology, 25, 241-243.

Palomba, D., Angrilli, A., \& Mini, A. (1997). Visual evoked potentials, heart rate responses and memory to emotional pictorial stimuli. International Journal of Psychophysiology, 27, 55-67.

Schupp, H. T., Cuthbert, B. N., Bradley, M. M., Birbaumer, N., \& Lang, P. J. (1997). Probe P3 and blinks: Two measures of affective startle modulation. Psychophysiology, 34, 1-6.

Schupp, H. T., Cuthbert, B. N., Bradley, M. M., Cacioppo, J. T., Ito, T., \& Lang, P. J. (2000). Affective picture processing: The late positive potential is modulated by motivational relevance. Psychophysiology, 37, 257-261. 
Schupp, H. T., Junghöfer, M., Weike, A. I., \& Hamm, A. O. (2003). Emotional facilitation of sensory processing in the visual cortex. Psychological Science, 14, 7-13.

Timberlake, W. (1993). Behavior systems and reinforcement: An integrative approach. Journal of the Experimental Analysis of Behavior, 60, 105-128.

Venables, P. H., \& Christie, M. J. (1980). Electrodermal activity. In I. Martin \& P. H. Venables (Eds.), Techniques in psychophysiology (pp. 3-67). Chichester, UK: Wiley. 